\title{
Is Endometrial Thickness on the Day of ET Really Predictive of IVF Outcome?
}

\author{
Richa Sharma, Kamini Rao, MS Srinivas, Theodre Jones
}

\begin{abstract}
Background: The effect of endometrial thickness on pregnancy rates in assisted reproductive technology (ART) patients has been evaluated by many authors, with controversial results. Endometrial thickness has been utilized as an indirect indicator for endometrial receptivity.
\end{abstract}

Objective: To evaluate relationship between endometrial thickness on day of embryo transfer and pregnancy outcome in in vitro fertilization and embryo transfer (IVF-ET) cycles. Should we cancel cycles based on endometrial thickness only?

Material and methods: A prospective analysis was conducted at Dr Kamini Rao Hospital, Bangaluru, of 239 patients. Various parameters were compared between pregnant and nonpregnant patients to see whether there is any cut-off for endometrial thickness on day of embryo transfer by which we can predict good prognosis in form of pregnancy and what effect other variables on endometrial thickness and pregnancy respectively and should we cancel embryo transfer, if endometrial thickness is not within certain range?

Results: In the study population, 174 (73\%) had primary and $65(27 \%)$ had secondary infertility, Ovarian stimulation was performed with long protocol in $37 \%$ cases, antagonist protocol in $47 \%$ and other protocols like microflare, short, ultralong, ultrashort in $15 \%$. Mean age of patients was $31.04 \pm 3.79$ years. Among causes of infertility male factor was present in $39 \%$, tubal factor was seen in $18 \%$, unexplained were $13 \%$, polycystic ovarian syndrome in $11 \%$, poor ovarian reserve in $4.1 \%$ and mixed causes in $13 \%$. Majority of our patients were in normal and overweight as per body mass index (BMI). ET were easy in $90 \%$ of cases and $14(5.8 \%)$ ETs were cancelled. The reason for cancellation was ovarian hyperstimulation syndrome (OHSS) in 9 cases, fluid in cavity in 2 cases, one patient had hyperpyrexia on day of ET and 2 cases of failed fertilization. Endometrial thickness was $>10 \mathrm{~mm}$ in $35 \%$ cases. Overall clinical pregnancy rate was $39 \%$ with implantation rate of $21 \%$, fertilization rate of $92 \%$ and cleavage rate of $95 \%$ and live birth rate of $26 \%$. There were more follicles, oocytes and embryos, the endometrium was $>10 \mathrm{~mm}$ and embryo quality was higher among women who became pregnant when compared with nonpregnant women after assisted reproduction though not statistically significant ( $p$ $>0.05$ ). The pregnancy rate improved as endometrial thickness increased showing a linear association.

Conclusion: Increased endometrial thickness is associated with higher pregnancy rates, but as such a cut off cannot be decided. In our study we have seen pregnancies at both thin and thick endometrium so we should not cancel ET merely on the basis of endometrial thickness as pregnancy is affected by multiple variables and not by endometrial thickness alone.

Date of Received: 18-07-12

Date of Acceptance: 14-08-12

Date of Publication: May 2012
Keywords: Endometrial thickness, Gonadotropin, In vitro fertilization, Pregnancy.

How to cite this article: Sharma R, Rao K, Srinivas MS, Jones T. Is Endometrial Thickness on the Day of ET Really Predictive of IVF Outcome? Int J Infertility Fetal Med 2012;3(2): 40-47.

\section{Source of support: Nil}

Conflict of interest: None declared

\section{INTRODUCTION}

Assisted reproductive technology (ART) has been commonly used in infertility treatment over the last two decades. The high cost and relatively low implantation and pregnancy rates (PRs) in in vitro fertilization (IVF) and intracytoplasmic sperm injection (ICSI) treatment cycles has led to a need to evaluate the predictors of success in these patients.

During the menstrual cycle the endometrium undergoes cyclic changes in preparation for implantation. In the follicular phase, the growing follicles produce increasing amounts of estradiol that will induce proliferative endometrial changes. ${ }^{10}$ Following ovulation, the corpus luteum produces progesterone that will initiate secretory changes. If implantation does not occur during the window of implantation, the endometrium will shed once the corpus luteum regresses. An association of various cycle characteristics and treatment outcome has been evaluated since the introduction of ARTs. According to its appearance in the longitudinal section, the endometrial morphologic characteristics can be classified as 'triple layer' or 'nontriple layer. ${ }^{20}$

One of the important factors is the endometrial receptivity. Endometrial thickness has been utilized as an indirect indicator for endometrial receptivity and is measured in the mid-saggital plane during transvaginal ultrasound, which is considered as both atraumatic and simple. ${ }^{1}$

The effect of endometrial thickness on pregnancy rates in ART patients has been evaluated by many authors. ${ }^{2-11}$ Some authors demonstrated a higher pregnancy rate at certain endometrial thickness, ${ }^{12-15}$ while others did not show a significant correlation between endometrial thickness and PRs in IVF/ICSI patients. ${ }^{5,7,8}$ Other authors reported a threshold of $<7 \mathrm{~mm}$ and/or $>14 \mathrm{~mm}$ with a significant 
reduction in implantation rate and $P R .{ }^{16,17}$ With these controversies, no conclusive cutoff value of endometrial thickness has been established in order to help clinicians in counseling the couple about the outcome. The reason for such controversy could be probably due to a relatively low number of cycles for patients with both extremes of endometrial thickness.

\section{MATERIALS AND METHODS}

It is a prospective clinical study of 239 patients performed at Jayanagar center, which is a unit of BACC (Bangalore Assisted Conception Centre), Bengaluru, Karnataka.

Inclusion criteria were fresh nondonor IVF cycles in age group of 25 to 35 years and patients with variable indications for IVF were enrolled.

Exclusion criteria were those with frozen embryo transfer (FET), donor oocyte or embryos, gestational surrogacy, having history of prior uterine surgery or Ashermann's syndrome, genital tuberculosis, congenital uterine or endometrial or myometrial anomalies and failed fertilization.

During the study period various stimulation protocols were used-long, antagonist and other protocols (ultralong/ short/ultrashort/microflare) depending on patient characteristics.

Various patient variables were taken in form of age, indication of IVF, baseline follicle stimulating hormone (FSH) level, type-fresh and number of cycle, body weight, height, body mass index (BMI), treatment protocol used, number of days of stimulation, number of ampules of gonadotropins used, type of gonadotropins, oocyte retrieval rate, fertilization rate, cleavage rate, endometrial thickness on day of embryo transfer which was performed on day 5 after human choronic gonadotropin (hCG) injection.

Various parameters were compared between pregnant and nonpregnant patients.

The pregnancy was accepted to be positive when betahCG level was over 50 IU on day 14 of ET. Clinical pregnancy was accepted when the embryo cardiac activity was observed by ultrasonography.

Their mean age was 32 years (range: 27 to 35 years). Infertility causes were male factor infertility in 93 cases, tubal factor infertility in 44 cases, unexplained in 33 cases, diminished ovarian reserve in 10 cases, ovulation disorders as PCOS in 27 cases, 8 case of endometriosis and other causes in 24 patients. About 229 (96\%) patients showed a normal basal FSH level ( $<10 \mathrm{mUI} / \mathrm{ml})$, had regular menstrual cycles. Approval for the study was obtained from the local institutional review board (IRB). Oral informed consent from the patients was required for this study, and was approved by the local ethics committee. In short, we performed ovarian stimulation with gonadotropins after pituitary suppression according to a long gonadotropinreleasing hormone (GnRH) protocol or antagonist protocol depending upon the patient parameters. Beginning with 150225 IU of recombinant FSH-GonalF (Serono, Italy) or Recagon (Organon) on a daily basis, the dose was adjusted according to follicular response and serum estradiol levels. In most cycles, purified human menopausal gonadotropin (hMG) was added (Menopur or hMG) to improve follicular growth. When a minimum of three dominant follicles greater than $18 \mathrm{~mm}$ were confirmed, 5,000 IU of hCG (Ovutrig HP, VHB Life Sciences, Mumbai, India) were administered.

The oocyte retrieval was performed 36 hours after the hCG injection. Oocytes were fertilized by IVF or ICSI, and the embryos were classified by grades (grade 1 for the best embryo) and given points through a cumulative embryo score. One to three embryos were transferred under ultrasound guidance. After the follicular puncture, all patients received $400 \mathrm{mg}$ of progesterone vaginally and luteal support was in form of estrogen and progesterone along with low dose Ecosprin and low molecular weight heparin (LMWH) in selected cases.

A pregnancy was confirmed by quantification of the serum hCG level 14 days after the ET. Values above 50 IU were considered positive. Ongoing gestation was defined as fetal heart activity at 12 weeks of gestation. A clinical pregnancy was defined by the presence of one or more gestational sacs with living embryos or alternatively by histologic confirmation of products of miscarriage.

\section{RESULTS}

Total number of patients-239, pregnant-87, nonpregnant - 138, number of cancelled cycles-14.

\section{DISCUSSION}

An association of various cycle characteristics and treatment outcome has been evaluated since, the introduction of ARTs. One such parameter, which has been evaluated by several groups, is that of endometrial thickness. ${ }^{3,18-21}$ Adequate proliferative and secretory changes are necessary for successful implantation to occur.

A triple-layer endometrial pattern and an endometrial thickness greater than $7 \mathrm{~mm}$ have been proposed as markers of endometrial receptivity but have yielded a high percentage of false-positive results. Uterine receptivity improves when blood flow increases under hormonal replacement therapy. The absence of a triplelayer pattern may be a sign of premature secretary changes indicating that the period of maximal receptivity may be over. Nonetheless, a pregnancy with a nontriple layer 
pattern is still possible so it cannot be sole indicator to cancel ET. ${ }^{12}$

The results of the present study identified a positive correlation in endometrial thickness between cycles that resulted in pregnancy and those that did not.

In our study, long protocol was used in 37\% patients, antagonist protocol in $47 \%$ cases and other protocols in $15 \%$ of patients. Mean age of $31.04 \pm 3.79$ years (Table 1 and Fig. 1). Among causes male factor in $36.4 \%$, tubal in $18 \%$, unexplained in $13 \%$, PCOS in $11 \%$ and mixed causes in $20 \%$ (Table 2). Majority (87\%) of patients fall in normal and overweight as per BMI (Table 3 and Fig. 2). Seventythree percent had primary and $27 \%$ had secondary infertility. Ninety percent ETs done were easy (Table 4) and 14 ETs cancelled in view of ovarian hyperstimulation syndrome (OHSS) in 9 cases and fluid in cavity in 2 cases, one patient had hyperpyrexia on day of ET and 2 cases of failed fertilization. Thirty-five percent had ET $>10 \mathrm{~mm}$ and overall clinical pregnancy rate was $39 \%$ with implantation rate of $21 \%$, fertilization rate of $92 \%$ and cleavage rate of $95 \%$ and live birth rate of $26 \%$. There were more follicles, oocytes and embryos, the endometrium was $>10 \mathrm{~mm}$ and embryo quality was higher among women who became pregnant when compared with nonpregnant women after assisted reproduction though not statistically significant ( $\mathrm{p} \geq 0.05$ ). The pregnancy rate improved as endometrial thickness increased showing a linear correlation.

In cycles that resulted in pregnancies, patients were younger, had more number of oocytes, more embryos transferred and the embryo quality was good. Also mean endometrial thickness on day of ET was higher in cases which got pregnant.

Adequate endometrial development is required for pregnancy to occur, and pregnancy rates were found to be higher when the endometrium reached at least $10 \mathrm{~mm}$ thickness. Consequently, clinicians providing IVF for infertile couples must pay close attention to endometrial development as well as to follicle growth.

In our study, we actually made analysis by dividing patients into two main groups:

- Group A with endometrial thickness $<10 \mathrm{~mm}$

- Group B with endometrial thickness $>10 \mathrm{~mm}$.

Results were analyzed in comparison between pregnant and nonpregnant patients.

In group A, pregnancy rate was $37 \%$ and in group B, pregnancy rate was $41 \%$ but outcome is statistically similar between two groups with $\mathrm{p}=0.514$ (Table 5).

We further subdivided these patients into eight subgroups depending on the endometrial thickness on the day of embryo transfer. Highest pregnancy of $34.5 \%$ was found in 9 to $10 \mathrm{~mm}$ group, $18.3 \%$ between 10 to $11 \mathrm{~mm}$, $16.1 \%$ in 11 to $12 \mathrm{~mm}$, up to $9.5 \%$ in 8 to $9 \mathrm{~mm}, 5.9 \%$ in 7 to $8 \mathrm{~mm}$ and $2.3 \%$ in those with endometrial thickness $>13 \mathrm{~mm}$. This linear correlation has been shown in figures. Thus, inference drawn was that higher endometrial thickness is positively associated with positive outcome with $\mathrm{p}=0.0 .166$ (Tables 6 and Fig. 2).

One pregnancy reported at $15 \mathrm{~mm}$ but turned to be missed abortion.

We had one pregnancy at $<7 \mathrm{~mm}(6.8 \mathrm{~mm})$ but unfortunately it turned out to be a missed abortion at 6 weeks gestation.

Besides we tried to see the affect of other variables (Tables 7, 8 and Figs 3 to 14) on pregnancy in comparison

\begin{tabular}{|lrr|}
\hline \multicolumn{3}{|c|}{ Table 1: Age distribution of patients studied } \\
\hline Age (years) & Number of subjects & $\%$ \\
\hline $21-25$ & 11 & 4.6 \\
$26-30$ & 123 & 51.5 \\
$31-35$ & 103 & 43.1 \\
$36-40$ & 2 & 0.8 \\
\hline Total & 239 & 100.0 \\
\hline
\end{tabular}

Mean \pm SD: $31.04 \pm 3.79$

\begin{tabular}{lcr}
\hline \multicolumn{2}{c}{ Table 2: Indication of infertility } \\
\hline Indication & Number of subjects & $\%$ \\
\hline Severe & 70 & 29.2 \\
oligoasthenoterato- & & \\
zoospermia & & \\
Tubal & 44 & 18.4 \\
Unexplained & 33 & 13.8 \\
Poor ovarian reserve & 10 & 4.1 \\
PCOS & 27 & 11.3 \\
Azoospermia & 23 & 9.6 \\
Mixed causes & 32 & 13.3 \\
\hline Total & 239 & 100.0 \\
\hline
\end{tabular}

\begin{tabular}{lcc}
\multicolumn{3}{|c}{ Table 3: BMI distribution of subjects studied } \\
\hline BMI $\left(\mathrm{kg} / \mathrm{m}^{2}\right)$ & Number of subjects & $\%$ \\
\hline$<18.5$ & 3 & 1.3 \\
$18.5-25.0$ & 84 & 35.1 \\
$25.0-30.0$ & 125 & 52.3 \\
$>30.0$ & 27 & 11.3 \\
\hline Total & 239 & 100.0 \\
\hline
\end{tabular}

Mean \pm SD: $25.68 \pm 3.81$

\begin{tabular}{lcr}
\multicolumn{3}{c}{ Table 4: Embryo transfer } \\
\hline ET & Number of subjects & $\%$ \\
\hline Easy & 214 & 89.5 \\
Difficult & 11 & 4.6 \\
ET cancelled & 14 & 5.8 \\
\hline Total & 239 & 100.0 \\
\hline
\end{tabular}


to the their effect on endometrial thickness. We found a nonsignificant correlation.

One group found that fecundity was increased when the endometrium was at least $9 \mathrm{~mm}$ thick, and had a triple-line appearance during IVF cycles. However, biochemical pregnancies were more frequent with a thinner endometrium. Others demonstrated an improved pregnancy rate with a thicker endometrium. ${ }^{10}$

Another group ${ }^{10}$ subsequently evaluated 516 IVF cycles and found pregnancy and ongoing pregnancy rates to be higher when the endometrial thickness was $>9 \mathrm{~mm}$. Likewise, a minimum thickness of $10 \mathrm{~mm}$ during IVF was found to produce a higher pregnancy rate.

The day of measurement might also influence the association between endometrial thickness and cycle outcome.

In the present study, an increased endometrial thickness was not related to improved pregnancy rates, although the measurements were made on the day of transfer-that is, at 4 or 5 days after the hCG injection. Endometrial thickness

\begin{tabular}{|c|c|c|c|}
\hline \multirow[t]{2}{*}{ Outcome } & \multicolumn{2}{|c|}{ Endometrial thickness ( $\mathrm{mm}$ ) } & \multirow[t]{2}{*}{ Total } \\
\hline & $<10.0$ & $>10.0$ & \\
\hline Negative & 90 (62.9\%) & 48 (58.5\%) & 138 (61.3\%) \\
\hline Positive & $53(37.1 \%)$ & $34(41.5 \%)$ & $87(38.7 \%)$ \\
\hline Total & $143(100.0 \%)$ & $82(100.0 \%)$ & 225 (100.0\%) \\
\hline
\end{tabular}

Inference: Outcome is statistically similar between two groups with $p=0.514$

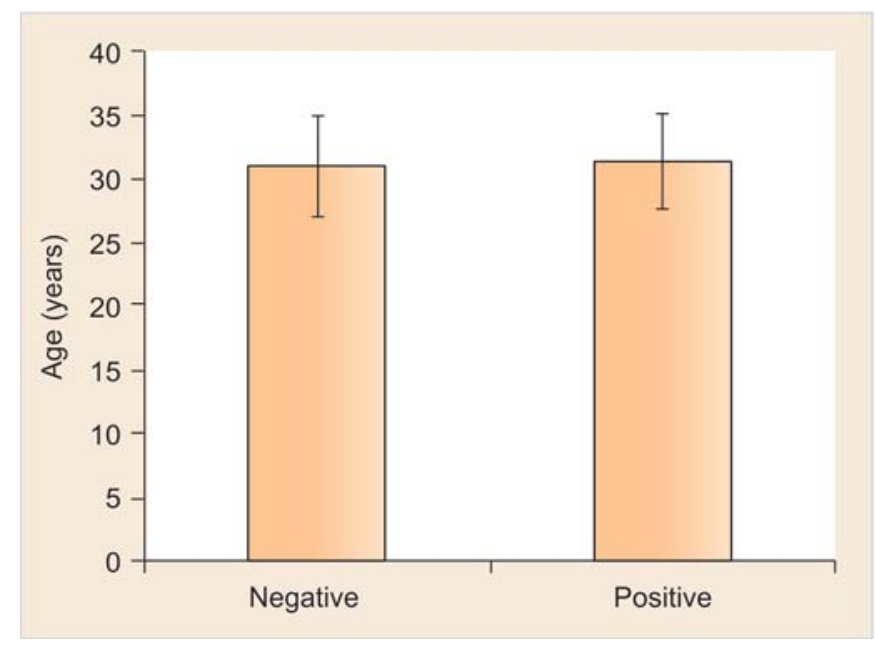

Fig. 1: Correlation between age and IVF outcome

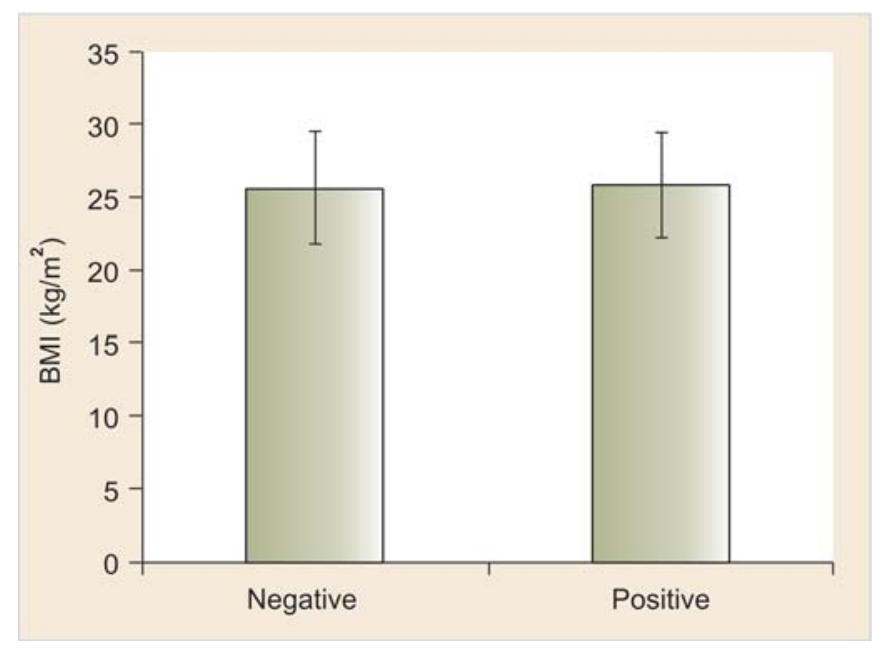

Fig. 2: Correlation between BMI and IVF outcome

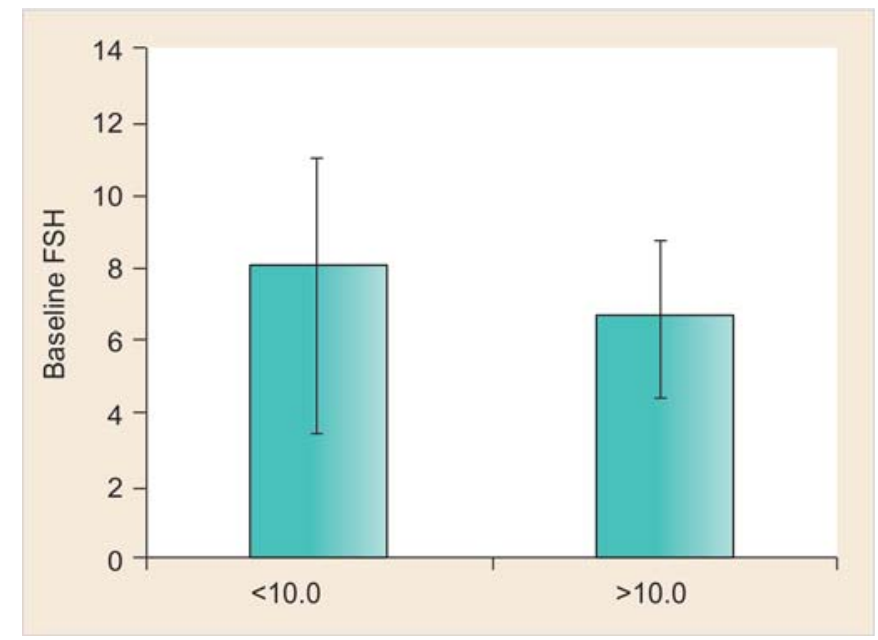

Fig. 3: Correlation between baseline FSH and endometrial thickness in two groups (A and $B$ )

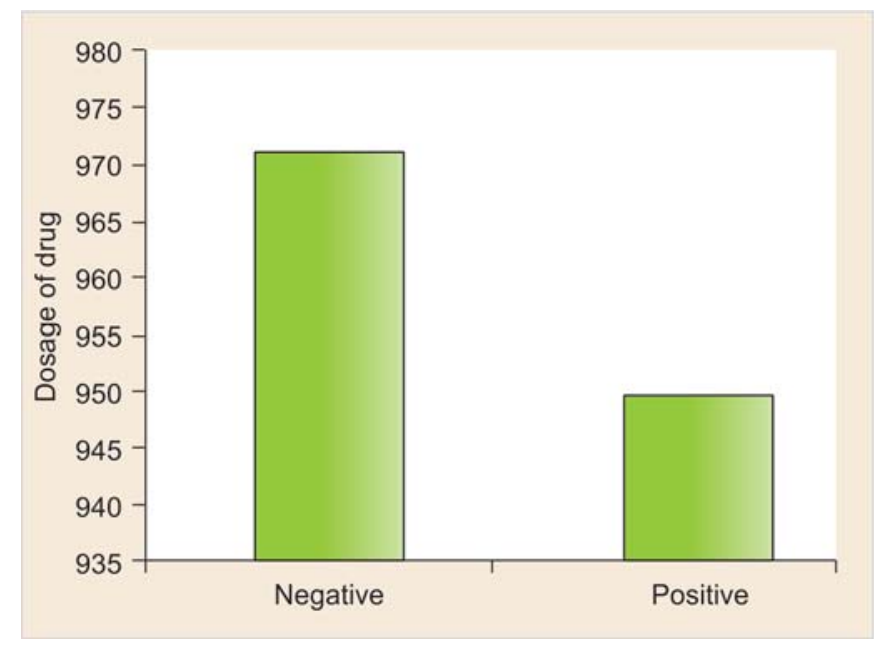

Fig. 4: Correlation between dosage of gonadotropins and IVF outcome 
evaluated on the day of embryo transfer might be influenced by an increased luteal phase progesterone secretion, and various measurement methods (including outer edge to outer edge, or outer edge to inner edge) could further affect outcome. Differences in the analysis might also provide a further explanation for the conflicting results.

Table 6: Comparison of endometrial thickness $(\mathrm{mm})$ with cycle outcome

\begin{tabular}{crr}
$\begin{array}{c}\text { Endometrial } \\
\text { thickness }(\mathrm{mm})\end{array}$ & \multicolumn{2}{c}{ Cycle outcome } \\
\cline { 2 - 3 } & $\begin{array}{r}\text { Negative } \\
(n=140)\end{array}$ & $\begin{array}{r}\text { Positive } \\
(n=87)\end{array}$ \\
\hline$<7$ & 0 & $1(1.1 \%)$ \\
$7-8$ & $14(10.0 \%)$ & $5(5.7 \%)$ \\
$8-9$ & $31(22.1 \%)$ & $17(9.5 \%)$ \\
$9-10$ & $46(32.9 \%)$ & $30(34.5 \%)$ \\
$10-11$ & $30(21.4 \%)$ & $16(18.3 \%)$ \\
$11-12$ & $16(11.4 \%)$ & $14(16.1 \%)$ \\
$12-13$ & $2(1.4 \%)$ & $2(2.3 \%)$ \\
13 and above & $1(0.7 \%)$ & $2(2.3 \%)$ \\
Mean \pm SD & $10.07 \pm 1.31$ & $10.32 \pm 1.39$
\end{tabular}

Inference: Higher endometrial thickness is positively associated with positive outcome with $p=0.166$
The effect of 'increased' endometrial thickness has also been evaluated. For example, one group ${ }^{4}$ reported lower implantation and pregnancy rates among women with an endometrial thickness $>14 \mathrm{~mm}$ on the day of hCG administration. However, no adverse effect of thickened (>14 mm) endometrium on implantation, pregnancy or abortion rates was identified by others. ${ }^{21}$ In our study, we found a direct relation between the endometrial thickness and the IVF outcome as we got pregnancies in 34\% cases with endometrial thickness between 9 and $10 \mathrm{~mm}$ on the day of ET.

Table 8: Comparison of BMI with cycle outcome

\begin{tabular}{ccc}
\hline \multirow{2}{*}{ BMI $\left(\mathrm{kg} / \mathrm{m}^{2}\right)$} & \multicolumn{2}{c}{ Cycle outcome } \\
\cline { 2 - 3 } & Negative $(n=140)$ & Positive $(n=87)$ \\
\hline$<18.5$ & $2(1.4 \%)$ & 0 \\
$18.5-25.0$ & $47(33.6 \%)$ & $32(36.8 \%)$ \\
$25.0-30.0$ & $76(54.3 \%)$ & $46(52.9 \%)$ \\
$>30.0$ & $15(10.7 \%)$ & $9(10.3 \%)$ \\
\hline
\end{tabular}

Inference: BMI is not statistically associated with cycle outcome with $p=0.699$

\begin{tabular}{lccc}
\multicolumn{2}{c}{ Table 7: Comparison of study variables according to outcome } \\
\cline { 1 - 2 } Study variables & \multicolumn{2}{c}{ Cycle outcome } & \multirow{2}{*}{$p$-value } \\
\cline { 2 - 3 } & Negative $(n=140)$ & Positive $(n=87)$ & 0.586 \\
Age in years & $30.92 \pm 3.89$ & $31.21 \pm 3.72$ & 0.755 \\
BMI $\left(\mathrm{kg} / \mathrm{m}^{2}\right)$ & $25.67 \pm 3.88$ & $25.84 \pm 3.62$ & 0.410 \\
Baseline FSH & $7.29 \pm 3.41$ & $7.74 \pm 4.77$ & 0.859 \\
Dosage of drug & $975.89 \pm 987.36$ & $949.62 \pm 892.58$ & 0.297 \\
Number of follicles $>14 \mathrm{~mm}$ & $10.50 \pm 6.18$ & $11.48 \pm 7.73$ & 0.201 \\
Mean no. of oocytes & $9.81 \pm 6.44$ & $11.05 \pm 7.84$ & 0.232 \\
Mean no. of M2 oocytes & $7.01 \pm 4.93$ & $7.87 \pm 5.78$ & 0.252 \\
Mean number of fertilized oocytes & $6.36 \pm 4.41$ & $7.10 \pm 5.26$ & 0.293 \\
Mean oocyte retrieval rate & $91.74 \pm 16.62$ & $93.92 \pm 11.85$ & 0.194 \\
Mean no. of embryos transferred & $2.45 \pm 0.82$ & $2.58 \pm 0.67$ & 0.210 \\
Number of embryos frozen & $3.98 \pm 2.99$ & $4.58 \pm 4.18$ & 0.166 \\
Endometrial thickness (mm) & $10.07 \pm 1.31$ & $10.33 \pm 1.39$ & 0.871 \\
Fertilization rate & $92.26 \pm 14.47$ & $92.57 \pm 11.76$ & 0.161 \\
Cleavage rate & $95.24 \pm 11.78$ & $97.32 \pm 7.59$ & \\
\hline
\end{tabular}

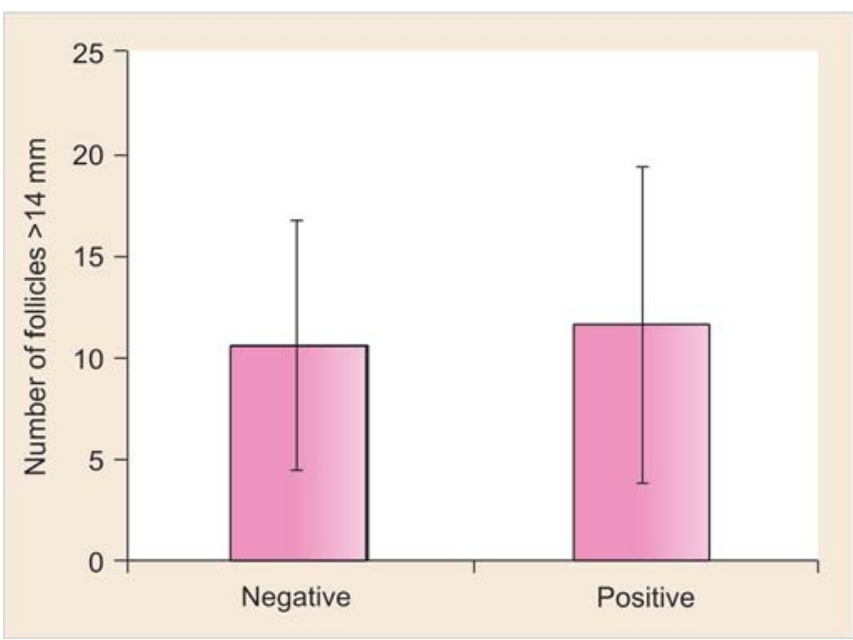

Fig. 5: Correlation between number of dominant follicles and IVF outcome

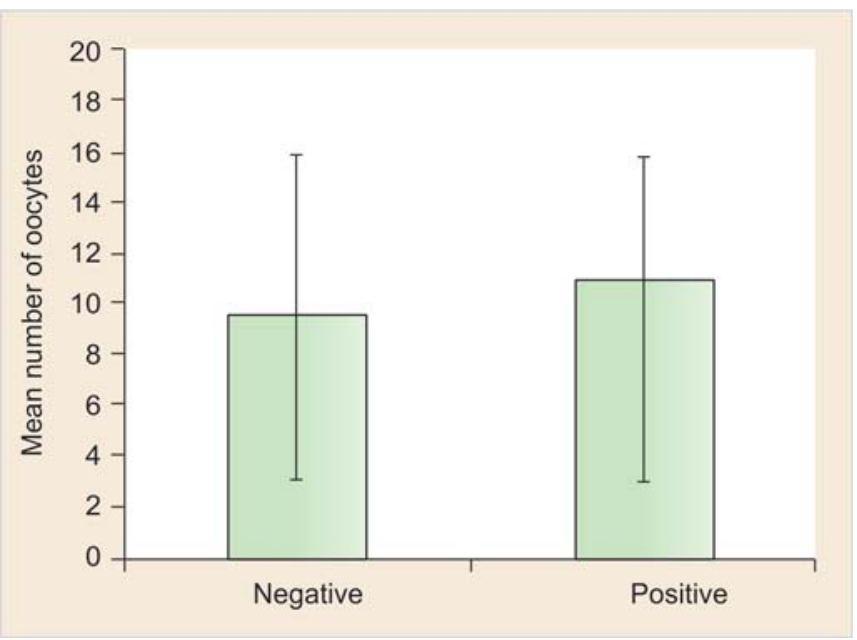

Fig. 6: Correlation between total number of oocytes and IVF outcome 


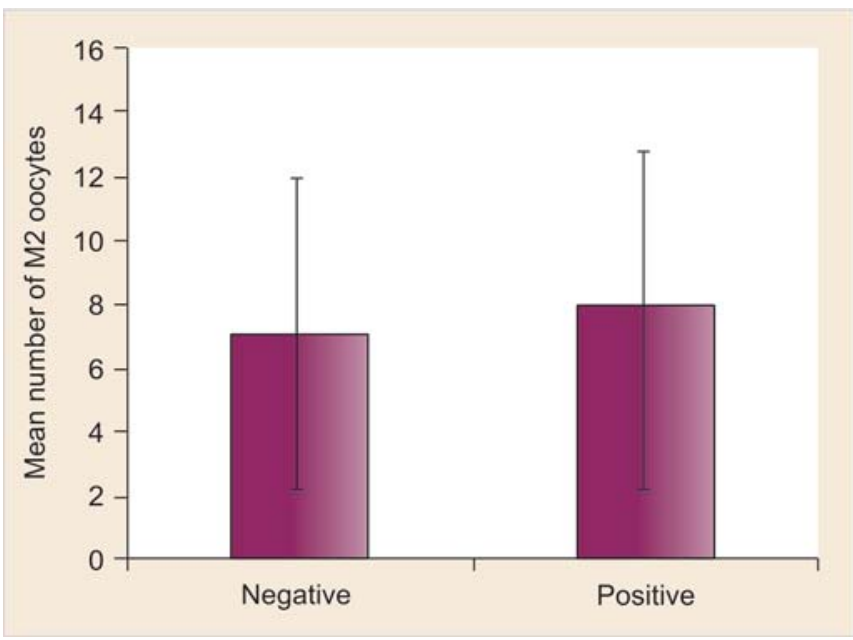

Fig. 7: Correlation between number of M2 oocytes and IVF outcome

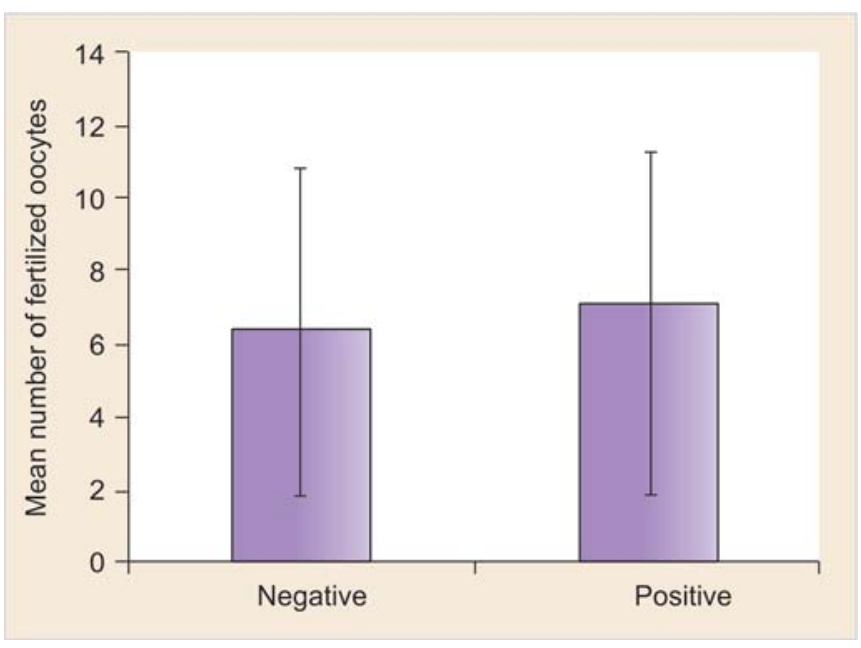

Fig. 8: Correlation between mean number of fertilized oocytes and IVF outcome

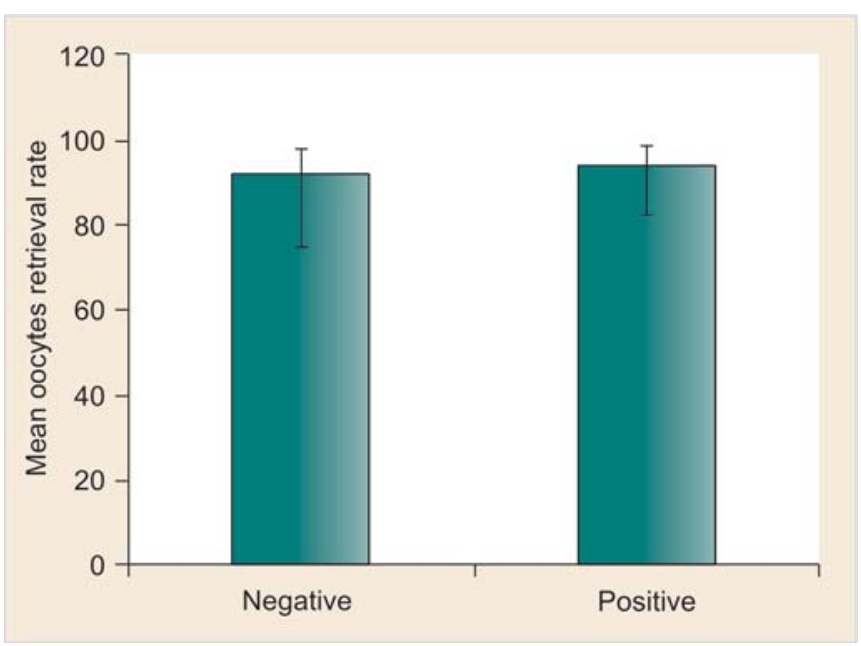

Fig. 9: Correlation between oocyte retrieval rate and IVF outcome

Adequate endometrial development is one of the factors that play a significant role in IVF outcome. Other variables, such as age, embryo quality, number of embryos transferred and stimulation protocol were also shown to have an

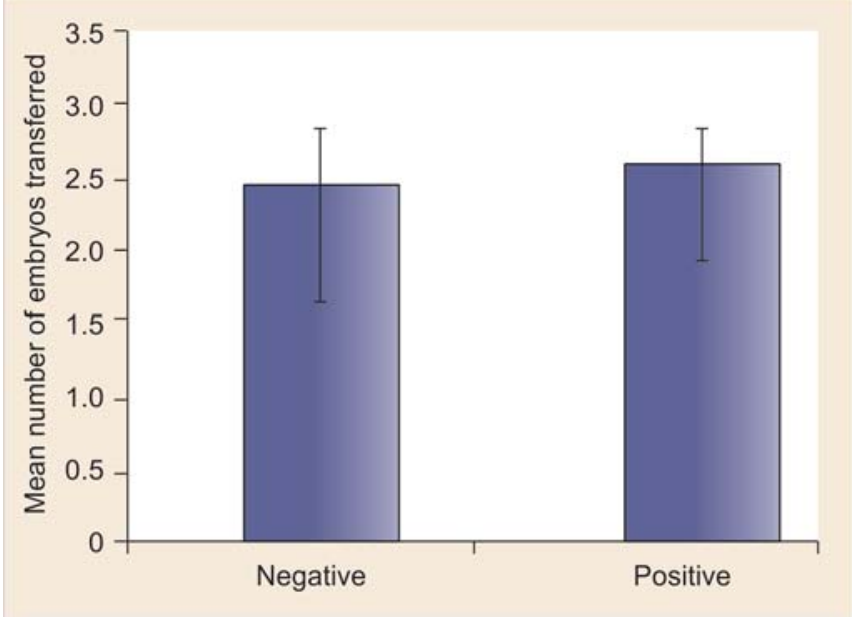

Fig. 10: Correlation between mean number of embryos transferred and IVF outcome

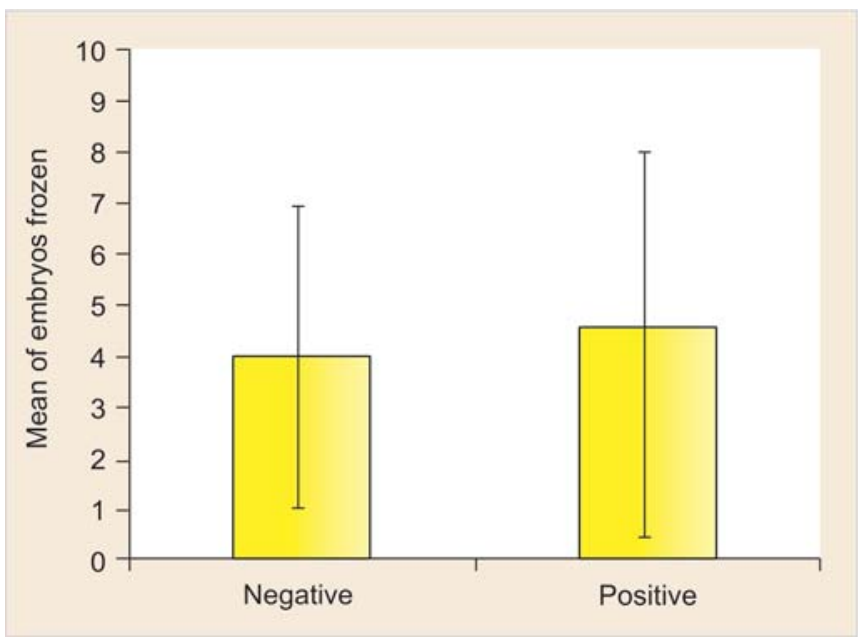

Fig. 11: Correlation between number of embryos frozen and IVF outcome

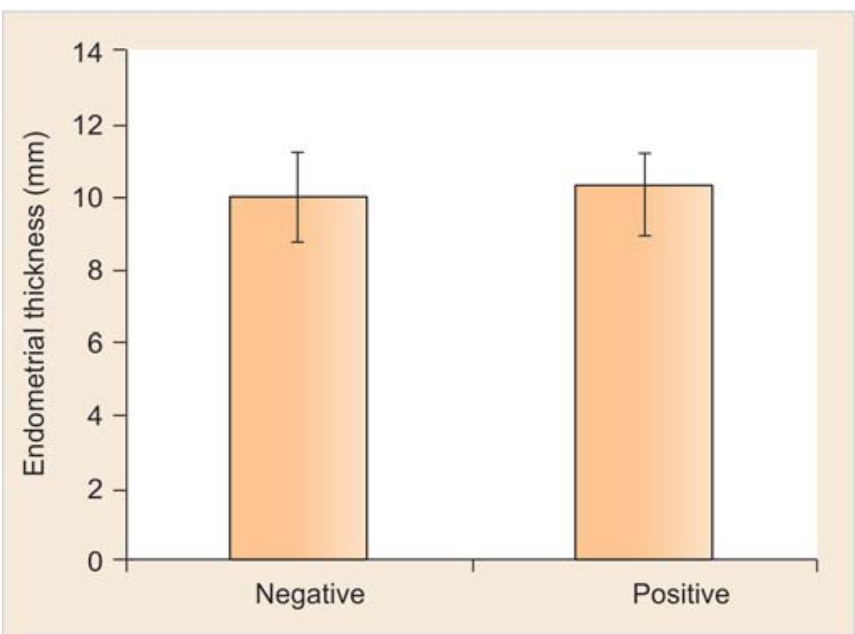

Fig. 12: Correlation between endometrial thickness and IVF outcome

impact on treatment outcome though not statistically significant.

From our study, we can conclude that pregnancy rate shows a linear correlation with increasing endometrial 


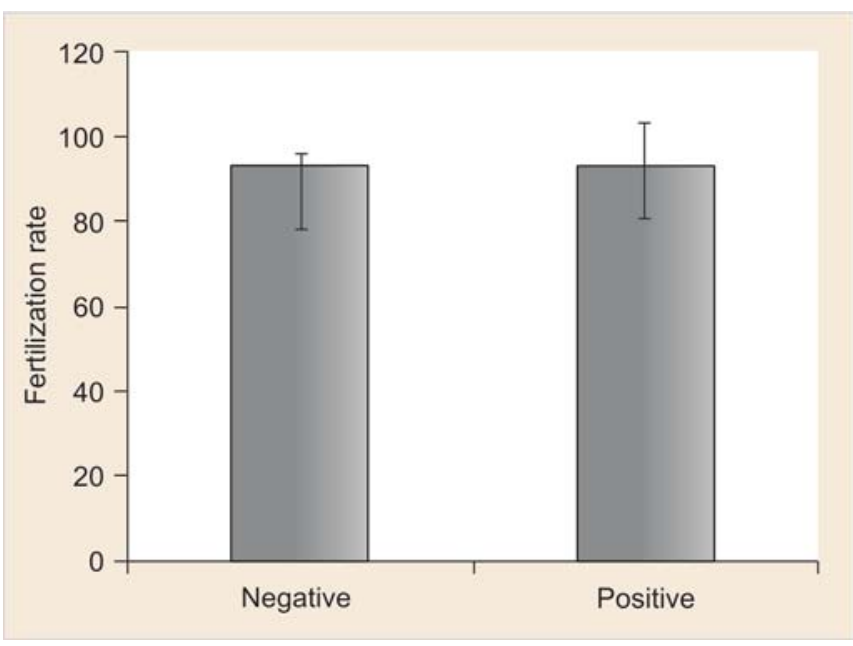

Fig. 13: Correlation between fertilization rate and IVF outcome

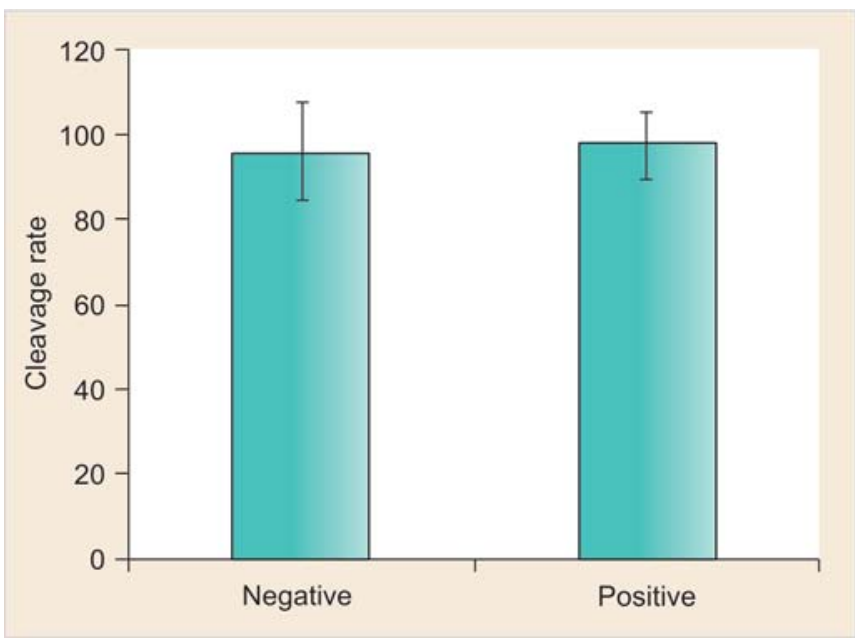

Fig. 14: Correlation between cleavage rate and IVF outcome

thickness but, as such cutoff for endometrial thickness cannot be decided. Thus, we are not justified in cancelling ET merely by keeping a cutoff in mind.

This is a prospective study and we are still continuing to analyse our results with a higher number.

\section{REFERENCES}

1. Freidler S, Schenker JG, Herman A, Lewin A. The role of ultrasonography in the endometrial receptivity following assisted reproduction treatmens: A critical review. Hum Reprod Update 1996;2:323-35.

2. Isaacs JD Jr, Wells CS, Williams DB, Odem RR, Gast MJ, Strickler RC. Endometrial thickness is a valid monitoring parameter in cycles of ovulation induction with menotropins alone. Fertil Steril 1996;65:262-66.

3. Noyes N, Liu HC, Sultan K, Schattman G, Rosenwaks Z. Endometrial thickness appears to be a significant factor in embryo implantation in in vitro fertilization. Hum Reprod 1995; 10:919-22.

4. Rinaldi L, Lisi F, Floccari A, Lisi R, Pepe G, Fishel S. Endometrial thickness as a predictor of pregnancy after in vitro fertilization but not after intracytoplasmic sperm injection. Hum Reprod 1996;11:1538-41.
5. Yuval Y, Lipitz S, Dor J, Achiron R. The relationships between endometrial thickness, and blood flow and pregnancy rates in invitro fertilization. Hum Reprod 1999;14:1067-71.

6. Weissman A, Gotlieb L, Casper RF. The detrimental effect of increased endometrial thickness on implantation and pregnancy rates and outcome in an in vitro fertilization program. Fertil Steril 1999;71:147-49.

7. De Geyter C, Schmitter M, De Geyter M, Nieschlag E, Holzgreve W, Schneider HP. Prospective evaluation of the ultrasound appearance of the endometrium in a cohort of 1,186 infertile women. Fertil Steril 2000;73:106-13.

8. Bassil S. Changes in endometrial thickness, thickness, length and pattern in predicting pregnancy outcome during ovarian stimulation in in vitro fertilization. Ultrasound Obstet Gynecol 2001;18:258-63.

9. Schild RL, Knobloch C, Dorn C, Fimmers R, Ven H van der, Hansmann M. Endometrial receptivity in an in vitro fertilization program as assessed by spiral artery blood flow, endometrial thickness, endometrial volume and uterine artery blood flow. Fertil Steril 2001;75:361-66.

10. Kovacs P, Matyas S, Boda K, Kaali SG. The effect of endometrial thickness on IVF/ICSI outcome. Hum Reprod 2003;18:2337-41.

11. Check JH, Nowroozi K, Choe J, Dietterich C. Influence of endometrial thickness and echo patterns on pregnancy rates during in vitro fertilization. Fertil Steril 1991;56:1173-75.

12. Dickey RP, Olar TT, Curole DN, Taylor SN, Rye PH. Endometrial pattern and thickness associated with pregnancy outcome after assisted reproduction technologies. Hum Reprod 1992; 7:418-21.

13. Gonen Y, Casper RF, Jacobson W, Blankier J. Endometrial thickness and growth during ovarian stimulation: A possible predictor of implantation in in vitro fertilization. Fertil Steril 1989;52:446-50.

14. Gonen Y, Casper RF. Prediction of implantation by the sonographic appearance of the endometrium during controlled ovarian stimulation for in vitro fertilization (IVF). J In Vitro Fert Embryo Transfer 1990;7:146-52.

15. McWilliams GD, Frattarelli JL. Changes in measured endometrial thickness predict in vitro fertilization success. Fertil Steril 2007;88:74-81.

16. Xingpi Z, Chi-Huang C, Edmond C, Randall B, Magdy M, Kazer RR. Increased endometrial thickness is associated with improved treatment outcome for selected patients undergoing in vitro fertilization-embryo transfer. Fertil Steril 2005;83: 336-40.

17. Check JH, Nowroozi K, Choe J, Lurie D, Dietterich C. The effect of endometrial thickness and echo pattern on in vitro fertilization outcome in donor oocyte-embryo transfer cycle. Fertil Steril 1993;59:72-75.

18. Yoeli R, Ashkenazi J, Orvieto R, Shelef M, Kaplan B, Bar-Hava I. Significance of increased endometrial thickness in assisted reproduction technology treatments. J Assist Reprod Genet 2004;21:285-89.

19. Quintero RB, Sharara FI, Milki AA. Successful pregnancies in the setting of exaggerated endometrial thickness. Fertil Steril 2004;82:215-17.

20. Luis T, A Maria J Barco, Santiago Bau, et al. Are endometrial parameters by three-dimensional ultrasound and power Doppler angiography related to in vitro fertilization/embryo transfer outcome? American Society for Reproductive Medicine. Fertil Steril_2008;89:111-17. 
21. Dietterich C, Check JH, Choe JK, Nazari A, Lurie D. Increased endometrial thickness on the day of human chorionic gonadotropin injection does not adversely affect pregnancy or implantation rates following in vitro fertilization-embryo transfer. Fertil Steril 2002;77:781-86.

\section{ABOUT THE AUTHORS}

\section{Richa Sharma (Corresponding Author)}

Fellow, Department of Reproductive Medicine, Bangalore Assisted Conception Centre, Bengaluru, Karnataka, India e-mail: richasharma47@gmail.com

\section{Kamini Rao}

Director, Department of Reproductive Medicine, Bangalore Assisted Conception Centre, Bengaluru, Karnataka, India

\section{MS Srinivas}

Chief Embryologist, Department of Embryology and Andrology Bangalore Assisted Conception Centre, Bengaluru, Karnataka India

\section{Theodre Jones}

Embryologist, Department of Embryology and Andrology, Bangalore Assisted Conception Centre, Bengaluru, Karnataka, India 